\title{
Approaching Intelligent Infection Diagnostics: Carbon Fibre Sensor for Electrochemical Pyocyanin Detection
}

\author{
Duncan Sharp ${ }^{1 \star}$, Robert Smith ${ }^{2}$, Stephen Forsythe ${ }^{1}$ and James Davis ${ }^{1}$ \\ 1. School of Science and Technology, Nottingham Trent University, Nottingham, NG11 8NS, UK \\ 2. Chemistry Dept. Faculty of Forensic and Investigative Sciences, University of Central Lancashire, Preston, PR1 2HE
}

*To whom correspondence should be addressed:

T: +44 (0) 115848 3218; E: duncan.sharp@ntu.ac.uk

Submitted to:

Biosensors and Bioelectronics 


\begin{abstract}
The pertinence of exploiting carbon fibre tow as an electrochemical sensing matrix for assessing pyocyanin production is evaluated. Pyocyanin is released by Ps. aeruginosa as a quorum signalling molecule during wound colonisation, increasing virulence and damaging host physiology, both contributing to an increased risk of infection. Prototype sensor assemblies have been developed and response characteristics towards pyocyanin are detailed. Sensitive and precise electrochemical measurements of pyocyanin by square wave voltammetry are established that enable the periodic monitoring of wound exudates for the presence of pyocyanin and serve as an early/pre- infection marker. As such, the pyocyanin sensor is presented as a possible sensor for intelligent wound management though incorporation into a 'smart-bandage' assembly or for use as a Point of Care Test for detection of Ps aeruginosa in cystic fibrosis patients' sputa.
\end{abstract}

\title{
Keywords:
}

Carbon fibre, smart bandage, pyocyanin, Pseudomonas aeruginosa, infection 


\section{Introduction}

The risk of hospital acquired (nosocomial) infections is relentless within modern healthcare. Whilst there are a multitude of factors that can give rise to infection in most cases however - the causative organisms are adventitious bacteria such as Pseudomonas aeruginosa, Staphylococcus aureus or Clostridium difficile [Mousa 1997, Chai et al 2000 and Vidhani et al 2001]. These have a direct impact on patient morbidity and mortality [Tredget et al 2004] and the associated complications that arise are estimated to increase the length of hospitalisation by between 6 and 13 days [Plowman 2000 and Fisher 1994]. Additionally, this incurs a substantial cost to healthcare, with a cost to the UK NHS alone in the billion pound region [Plowman 2003]. Whilst patient care guidelines from bodies such as the World Health Organisation (WHO) and the Health Protection Agency (HPA) are important, technology also has a part to play and recent advances in nano-particle science have seen the development of antibacterial dressings, frequently silver based [Atiyeh et al 2007]. Nevertheless, the innate adaptability of bacteria to rapidly mutate and generate resistant strains signifies the need for failsafe systems to identify the advent of a potential infection.

The opportunistic pathogen Ps. aeruginosa is the cause of a variety of infections, with compromised patients being particularly susceptible targets. Amongst the most widespread of which are pulmonary infection in cystic fibrosis patients and wound infections. Infection of burns wounds are a particular problem, due to their physiology, increasing both morbidity and mortality [Tredget et al 2004]. This 
coupled with the high incidence of antibiotic-resistant Ps. aeruginosa strains [Pirnay et al 2003, Cardo et al 2004] prompts the necessity to develop new antibiotic strategies. The ability to detect a $P$ s. aeruginosa infection early could not only improve patient morbidity and mortality, but reduce the time spent in hospital, reduce patients' pharmaceutical requirements and help curtail the financial burden of wound infections.

Ps. aeruginosa uses many complex quorum sensing (communication) systems: regulatory mechanisms enabling bacteria to make collective decisions with respect to the expression of a specific gene set [Rasmussen and Givskov 2006] controlling virulence. Quorum sensing systems function through the release of sensing molecules, including: elastase, alkaline phosphatase, exotoxin A, secretion proteins, catalase, rhamnolipid, lectins, acylated homoserine lactones, superoxide dismutase and pyocyanin. [Smith and Iglewski 2003]. The quorum sensing components have been found to directly effect the virulence [Smith and Iglewski 2003] and the host's response to the infection. Pyocyanin (1-hydroxy- $N$ methylphenazine) is a blue redox-active phenazine dye synthesized de-novo by 96-98\% of Pseudomonas aeruginosa strains [Reyes et al 1982] as part of the quorum sensing systems. Due to the low molecular weight of pyocyanin [Watson et al 1986], it is readily diffusible and can permeate cell membranes. Upon entry into cells pyocyanin is reduced by $\mathrm{NAD}(\mathrm{P}) \mathrm{H}$ and may subsequently reduce molecular oxygen to superoxide, which in turn dismutates to hydrogen peroxide, 
thereby inducing oxidative stress in endothelial and epithelial cells. [Muller 2006, Muller 2002, O'Malley et al 2004]

The direct physiological effects on both epithelial and endothelial mammalian cells are of importance not only for its virulence determination within the Ps aeruginosa quorum sensing systems, but also for the direct affects (oxidative or not) on human physiology which may allow more serious infections to initiate. The physiological effects facilitating the progression of infection include: disruption of ciliary beating [Kanthankumar et al 1993], arresting cell growth and inducing apoptosis [Muller 2006], antioxidant depletion and oxidative stress [Muller 2002, O'Malley et al 2004, Lau et al 2004], acceleration of neutrophils apoptosis and reduced inflammatory response [Usher et al 2002] and bacterial resistance to innate immune system [Schaber et al 2004]. Pyocyanin acts antimicrobially towards other bacteria by oxidative means, (e.g. against $E$. coli), whereas Ps. aeruginosa itself appears immune to the oxidative effects of the pyocyanin by having limited redox-cycling [Hassett et al 1992].

Whilst it is impossible to establish a reference range for pyocyanin production through Ps. aeruginosa infection, the concentration range of $0-100 \mu \mathrm{M}$ has been investigated as this covers the concentrations liable to be encountered [Muller 2006, Muller 2002, O'Malley et al 2004] and the diagnostically relevant range that has been observed in biofluids [Wilson et al 1988]. 
The foundations of the pyocyanin sensor reported here rely upon the properties of carbon fibre tow allowing use as combined detection element and transduction conduit.

The challenge and objective of the current investigation is the development of a sensor suitable for the detection of pyocyanin within biofluids, relevant to a variety of biomedical applications. Whilst the rudimentary detection of pyocyanin in biofluids has been studied at Hanging Mercury Drop Electrodes (HMDEs) [Vukomanovic et al 1996] its detection in wound fluid at solid electrodes within a Point of Care Testing format has yet to be considered. A principal application of a miniaturised pyocyanin sensor would be in the form of a 'smart-bandage' to allow intelligent in-situ wound monitoring. The periodical in-situ analysis of wound exudates would allow the early detection of pyocyanin production thereby alerting the patient or clinical staff to the likelihood of progression from contamination to colonisation. It would also allow early instigation of antibiotic therapies as the infection initiates and this may be prior to the appearance of an infection.

The core rationale is outlined in Figure $\mathbf{1}$ whereby the carbon fibre tow is thermally sandwiched between polyester laminate, as detailed in previous publications [Sharp and Davis 2008x2]. The underlying carbon is selectively exposed to function as the sensing element by laser etching through the encapsulating sheet. 


\section{Experimental}

Materials \& Methods: All reagents were of the highest grade available and used without further purification unless otherwise stated. All analytical solutions were prepared using Britton-Robinson buffer (acetic, boric and phosphoric acids each at a concentration of $0.04 \mathrm{M}$ ) in deionised water (Elgastat, Elga UK) adjusted to $\mathrm{pH} 7.0$ (accumet $($ AP72, Fisher scientific) through the addition of sodium hydroxide (Sigma-Aldrich). Where required, samples were bubbled with oxygen-free nitrogen (BOC gases) for 5 minutes to remove oxygen or spiked with folic acid / folate (Sigma-Aldrich) prior to analysis.

Pyocyanin synthesis and purification: Pyocyanin synthesis was performed by the photochemical degradation of $0.5 \mathrm{~g} / \mathrm{L}$ phenazine methosulfate, PMS, (SigmaAldrich) [as used by Knight et al 1979] in Britton-Robinson buffer ( $\mathrm{pH} 7.0)$. The synthesis was performed in a photochemical reactor (Figure 2) consisting of $8 \mathrm{~m}$ of HPLC grade $1.5 \mathrm{~mm}$ I.D. clear tubing wound around a commercially available aluminium fluorescent light fitting (Kengo lighting TCF13, 13W lamp) and wrapped in tin foil to increase light-PMS interaction, PMS solution was circulated for 6 hours at $5 \mathrm{~mL} / \mathrm{min}$ using a peristaltic pump (Gilson MINIPULS3). This yielded a strong blue coloured solution, from which the crude pyocyanin was extracted in three volumes of chloroform (Fisher Scientific), dried with magnesium sulphate (Fisher Scientific) and filtered (Whatman No. 42) prior to rotor-evaporation, in a $70^{\circ} \mathrm{C}$ waterbath, under vacuum onto chromatography silica gel (40-63u 60A FLUOROCHEM). The crude pyocyanin could then be 
purified by column chromatography in a $50 \times 5 \mathrm{~cm}$ column using 15:85 methanol:

chloroform, collecting only the blue portion and rotary-evaporated to dryness under vacuum. Purity was then ascertained by silica TLC plates (Rf 55-63 crescent using eluent), melting point determination $\left(M P=135^{\circ} \mathrm{C}\right)$ (stuart $\circledast \mathrm{SMP} 10$ ) and NMR. The novel use of this photochemical reactor setup facilitated $\sim 40 \%$ yield in 6 hours, allowing the synthesis and purification within a single working day.

Sensor Construction: Laminated carbon fibre tow electrodes were prepared by thermally sandwiching Carbon Fibre tow, 2000x $10 \mu \mathrm{m}$ diameter filaments (Goodfellow, UK) between $75 \mu \mathrm{m}$ thick sleeves of laser-etched (FB400 series CadCam Technology Ltd, Nottingham, UK) lamination pouch (Rexel UK) using a commercially available laminator. Electrical connection to the carbon tow was made through the presence of a strip of $100 \mu \mathrm{m}$ thick, adhesive-backed copper shielding tape (RS electronics). The electrodes were baked in a $100^{\circ} \mathrm{C}$ oven for 16 hours to ensure the complete permeation of the resin between the fibres within the laminate. This is necessary to ensure the mechanical integrity and coherence of the seal between the sensing fibre layer and the insulating polyester sheath such that no solvent creep or de-lamination wound occur during extended monitoring periods (e.g. 20 scans over 40 minutes). Sensor anodisation was performed in $0.1 \mathrm{M} \mathrm{NaOH}$ (Sigma) at $+2.0 \mathrm{~V}$ for 10 minutes.

Instrumentation: Electrochemical measurements were conducted using $\mu$ Autolab type III computer controlled potentiostat (Eco-Chemie, Utrecht, The 
Netherlands) using a three electrode configuration consisting of a Glassy Carbon Electrode (3mm diameter; BAS Technicol. UK) or Carbon Fibre tow laminate working electrode, a $3 \mathrm{M} \mathrm{KCl} \mathrm{Ag} \mid \mathrm{AgCl}$ half cell reference electrode (BAS Technicol. UK) and platinum wire counter electrode. Photometric measurements were performed using a JENWAY 6715 UV/Vis. spectrophotometer at $690 \mathrm{~nm}$. 


\section{Results and Discussion}

To allow the development of pyocyanin detection strategies the availability of pyocyanin is essential. The limited commercial availability of pyocyanin and high associated cost has lead to the purification or synthesis of pyocyanin by other means. Whilst pyocyanin has been extracted from bacterial cultures [Watson 1986, Cox 1986, Hassett et al 1992, Frank and DeMoss 1959] the low yield and extensive extraction steps required to remove all other bacterial metabolites are unfavourable. The most commonly used, photochemical oxidation of phenazine methosulfate (PMS), originated by Mcllwain in 1937 [Mcllwain 1937], consisted of an open flask of PMS in water $(1 \mathrm{~g} / \mathrm{L})$ exposed to sunlight until no further pyocyanin was produced, typically 'about 1 day'. A latter optimised version of this method used illumination by high intensity cool white fluorescent lights for 4 days at a PMS starting concentration of $0.5 \mathrm{~g} / \mathrm{L}$ (Knight et al 1979) and has been favoured since [Usher et al 2002]. The main disadvantage of both methods, but more so of the revised method, is the prolonged illumination period. Not only does this prevent rapid and upon-demand synthesis of pyocyanin, but the synthesised pyocyanin in solution may degrade to 1-hydroxyphenazine [Mcllwain 1937] affectively decreasing the efficiency. Increasing the photochemical reaction efficacy by using a photochemical reactor, as described within, allows a dramatic reduction in the duration of the photo-oxidation step allowing synthesis and purification within a single working day. 
The preliminary assessment of carbon fibre substrates enabled comparison with the commonly used glassy carbon electrode voltammetry (Figure 3). Additionally, Figure 3, facilitated the comparison of plain (un-modified) and anodised (modified) carbon fibre tow, anodised

Figure 3. Comparison of electrochemical detection of Pyocyanin by square voltammetry using GCE, plain and anodised carbon fibre sensors

substrate has proven more adept to the quantification of certain biomarkers e.g. urate [Sharp and Davis 2008]. The anodised tow has a substantially greater response at lower concentrations, due to the anodic fracturing previously

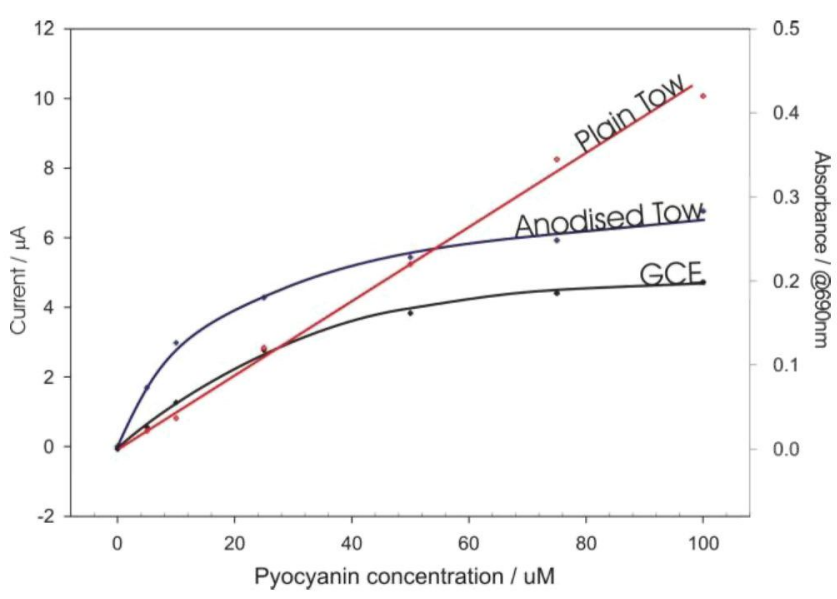
observed following anodisation: increasing electron transfer kinetics due to the presence of more edge plan sites and greater concentration of oxygen species [Tao et al 2007]. As observed when using the glassy carbon electrode, the response is clearly non-linear as a result of the modifications and is saturated at much lower concentration than those required to be quantified in a biomedical scenario, as outlined in the introduction and is therefore unsuitable. 
Figure 4. Square wave volammograms detailing the Pyocyanin oxidation peaks observed using calibration standards

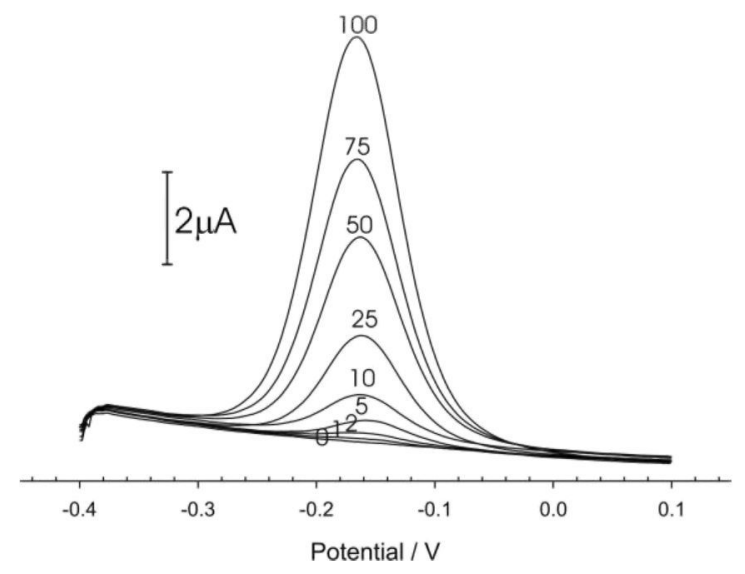

Plain carbon fibre tow does provide a linear response up to $100 \mu \mathrm{M}$ and was thus subject to further evaluation. Square wave voltammetry using plain carbon fibre tow gave rise to a single sharp oxidation peak observed at $-0.17 \mathrm{~V}$ which is attributed solely to the oxidation of pyocyanin (Figure 4). The scan range used $(-0.4 \mathrm{~V}$ to $+0.1 \mathrm{~V}$ ) was chosen specifically to prevent the polymerisation of pyocyanin onto the electrode at higher potentials as illustrated by cyclic voltammetry (Figure 5). The phenolic oxidation of pyocyanin ( $\mathrm{I}$ ) at $+0.85 \mathrm{~V}$ is responsible for the polymerisation of pyocyanin. The increase in pyocyanin oxidation and reduction peaks with increasing scans is the

Figure 5. Cyclic voltammograms of pyocyanin after 0 , 10,20 and 30 scans.

result of 2 electron transfer between the two polymeric forms (II) and (III) at $-0.18 \mathrm{~V}$ and $-0.25 \mathrm{~V}$, respectively, with increasing scans.

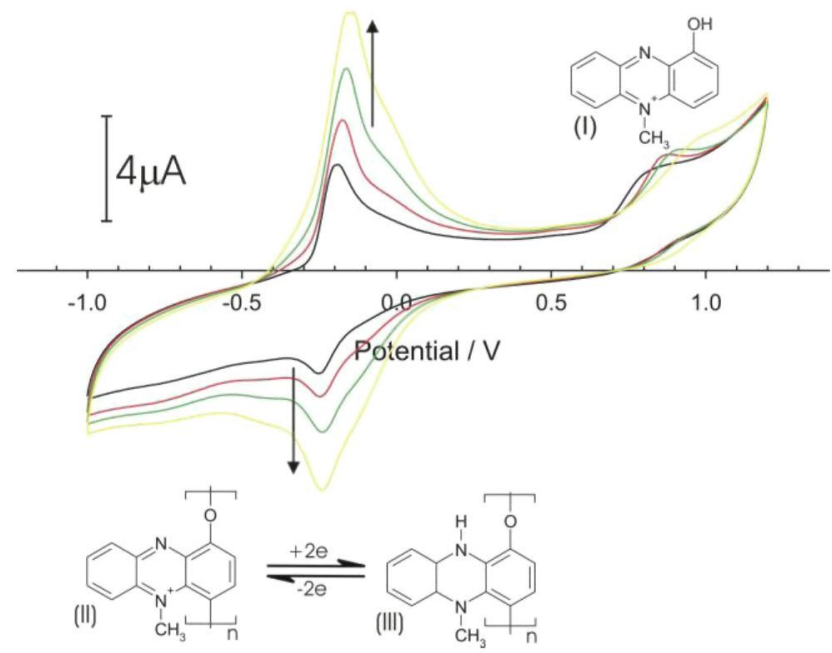


Calibrations were performed in triplicate using a wide range of pyocyanin standards $(1-100 \mu \mathrm{M})$, to ensure accuracy and precision across the necessary biomedically important range. The designed electrode composition enabled linear quantification from $1 \mu \mathrm{M}$ to $100 \mu \mathrm{M}$ in buffered solutions (peak height $/ \mu \mathrm{A}=$ 0.0814 [Pyocyanin conc. $/ \mu \mathrm{M}]-0.015, n=9, R^{2}=0.998$ ) indicating suitability for the proposed applications. The limit of detection of $0.030 \mu \mathrm{M}(\mathrm{LOD}=3 \mathrm{~s} / \mathrm{b})$ is beneficial to biomedical applications as sensitive detection of pyocyanin would enable the early detection of quorum sensing production of pyocyanin, by colonising PS. aeruginosa. The earlier an infection can be diagnosed and treated leads to improved recovery rates and reduced chance of resistant strains of PS. aeruginosa emerging. Furthermore, whilst relatively high concentrations of pyocyanin can be observed visually, by the strong blue colour of the pyocyanin pigment, this may be masked by the presence of blood in e.g. wound exudates and concentrations below $10 \mu \mathrm{M}$ appear colourless to the eye and subjective. This sensor would be able to detect at such low concentrations.

Given that the sensor can clearly detect pyocyanin, a subsequent issue to be addressed relates to whether the sensor can reliably measure the pyocyanin concentration beyond the first three measurements used for calibration and assessment of linearity. Whilst this may not be an issue for certain applications e.g. single scan outpatient detection of pyocyanin in sputa of cystic fibrosis patients, it is for the applications where continuous sampling is required. The use 
of a pyocyanin sensor within a wound environment would require periodic scanning to allow the early detection of pyocyanin. Two concentrations (10 and $50 \mu \mathrm{M})$ were used for intra-batch precision assessment, through 20 periodic replicates. The pyocyanin oxidation results in clearly visible, sharp peaks (Figure 6 inlay) with no drift in oxidation potential with increasing replicates.

After the requisite equilibration / preactivation scans (not shown), the height of each pyocyanin oxidation peak is presented as a percentage change from the mean (Figure 6). The coefficient of variance for the $10 \mu \mathrm{M}$ and $50 \mu \mathrm{M}$ populations

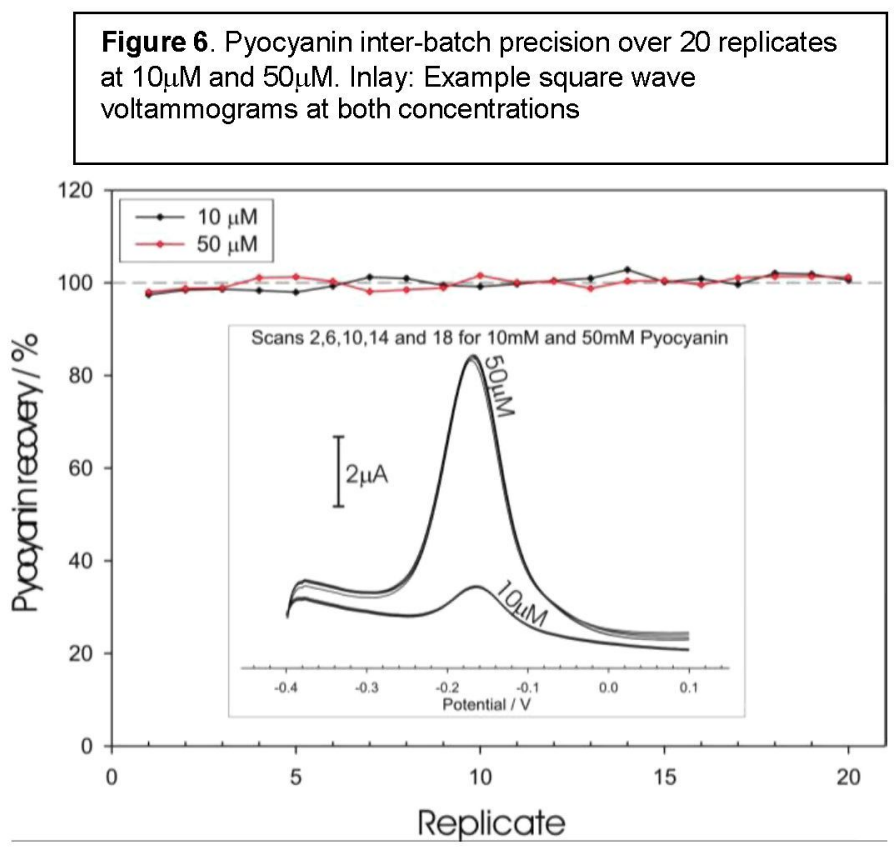
are $1.2 \%$ and $1.4 \%$ respectively, with no deviation greater than $2.6 \%$ for either concentration, thus showing the narrow distribution of oxidation peaks. The graphical representation highlights the absence of substantial changes or obvious trends in oxidation peak height, effectively showing the absence of pyocyanin polymerisation or electrode fouling by oxidised pyocyanin metabolites.

Due to the potential variety of biomedical applications for this novel pyocyanin sensor and the facultative anaerobe nature of Ps. aeruginosa it is important ensure the measurement is not affected by the presence or absence of molecular 
oxygen. The measurement of a series of standards in triplicate performed both normally aerated and following bubbling with oxygen-free nitrogen gas, lead to the formation of two linear (Oxygen present: Peak height $/ \mu \mathrm{A}=0.0764[$ Pyocyanin conc. $/ \mu \mathrm{M}]+0.0378, \mathrm{n}=7, \mathrm{R}^{2}=0.999$ and oxygen absent: Peak height $/ \mu \mathrm{A}=$ $0.0779[$ Pyocyanin conc. $/ \mu \mathrm{M}]+0.0961, \mathrm{n}=7, \mathrm{R}^{2}=0.998$ ) calibration plots. Whilst these are important as separate entities, the comparison of the two shows minimal difference between aerobic and anaerobic electrochemical detection of pyocyanin, again indicating suitability for use in a variety of applications.

The negative potentials and narrow scan range utilised for the quantification of pyocyanin limits the potentially interfering the affects of $1 \mu \mathrm{M}$ and $10 \mu \mathrm{M}$ folate additions on pyocyanin oxidation.

endogenous molecules. Folate is the only biomarker considered electroactive within this potential range at an appreciable concentration in biofluids, (reference range of up to $0.045 \mu \mathrm{M}$ in adult serum [Burtis et al 2006]) therefore this was the only analyte deemed necessary to investigate for interference. Figure 7 shows that even greater folate concentrations have no observable effect on the pyocyanin oxidation peak of a $10 \mu \mathrm{M}$ pyocyanin standard, even at equimolar concentrations (over 200x the top of the adult folate reference range). The applicability of a sensitive pyocyanin sensor is not limited to that of a 'smart-bandage' within a biomedical context. Pyocyanin concentrations within 
cystic fibrosis (CF) patients' sputa have been detected as high as $130 \mu \mathrm{M}$ [Wilson et al 1988]. The aforementioned affects on neutrophil apoptosis may be a clinically important mechanism of persistent infections by $P S$ aeruginosa in human tissue (e.g. chronic infections in CF patients) [Usher et al 2002]. Due to the potential severity and associated problems of $P$ s. aeruginosa infection within CF patients, a point of care testing (POCT) device enabling self/home monitoring of pyocyanin within the patient sputa could enable early detection of lung colonisation and by enabling earlier treatment of the patient during the infection may help the patients outcome and survival. An important factor to consider regarding pyocyanin as an infection biomarker is that pyocyanin is released prior to virulent colonisation, consequently it may be possible to detect the progression towards infection before a clinical infection is apparent.

\section{Conclusions}

The assessment of carbon fibre tow for the suitability of pyocyanin detection has allowed the interference free, sensitive and precise detection of pyocyanin at biologically relevant concentration. The sensor detailed within is thus deemed as potentially suitable for encompassment into a 'smart-bandage' to allow the early detection of Ps. aeruginosa colonisation of wounds. The sensor is also suggested as a potential Point of Care Test for the early detection and monitoring of pulmonary infections in cystic fibrosis patients.

\section{Acknowledgements}


The authors thank the EPSRC for supporting this work.

\section{References:}

Aqui, L., Guzman, A., Yanez-Sedeno, P. and Pingarron, J.M. 2002 Analytica Chimica Acta 461, 65-73

Atiyeh, B.S., Costagiola, M., Hayek, S.N. and Dibo, S.A. 2007 Burns 33, 139-148

Burtis, C.A., Ashwood, E.R., Bruns, D.E. 2006 Tietz Textbook of Clinical Chemistry and Molecular Diagnostics $4^{\text {th }}$ Edition, Elsevier Saunders, Missouri, USA.

Cardo, D., Horan, T., Andrus, M., Dembinski, M., Edwards, J., Peavy, G., Tolson, J. and Wagner, D. 2004 AJIC special article - National Nosocomial Infection Surveillance System Report.

Chai, J., Sheng, Z., Yang, H., Diao, L. and Li, L. 2000 Chinese Medical Journal 113, 1142-1146

Cox, C.D. 1986 Infection and Immunity 263-270

Crespi, F. 1996 Biosensors and Bioelectronics 11, 743-749

Dressman, S.F., Peters, R.L. and Micheal, A.C. 2002 Journal of Neuroscience Methods 119, 7581

Dutt, J.S.N., Livingstone, C., Cardosi, M.F., Wilkins, S.J. and Davis, J. 2006 Talanta 68, 14631468

Ezekiel, H.B., Sharp, D., Marti Villalba, M. ansd Davis, J. 2008 Journal of Chemistry and Physics of Solids 69, 2932-2935

Fisher, J. 1994 The Plague Makers, Simon and Schuster, New York USA

Frank, L.H. and DeMoss, R.D. 1959 Journal of Bacteriology 77, 776-782

Hassett, D.J., Charniga, L., Bean, K., Ohman, D.E. and Cohen, M.S. 1992 Infection and Immunity $60328-336$

Ju, H.X., Dong, L. and Chen, H.Y. 1996 Talanta 43, 1177-1183

Ju, H., Sun, H. and Chen, H. 1996 Analytica Chimica Acta 327, 125-132

Kanthankumar, K., Taylor, G., Tsang, K.W.T., Cundell, D.R., Rutman, A., Smith, S., Jefferey, P.K., Cole, P.J. and Wilson, R. 1993 Infection and Immunity 2848-2853

Katrlic, J. and Zaleskova, P. 2002 Bioelectrochemistry 56, 73-76

Knight, M., Hartman, P.E., Hartman, Z., Young, V.M.A. 1997 Analytical Biochemistry 95, 19-23

Lau, G.W., Hassett, D.J., Ran, H. and Kong, F. 2004 Trends in Molecular Microbiology 10, 599606

Liu, D. and Jin, W. 2003 Journal of Chromatography B 789, 411-415

Logman, M.J., Budygim, E.A., Gainetdinov, R.R. and Wightmanm R.M. 2000 Journal of Neuroscience Methods 95, 95-102 
Mcllwain, H. 1937, Journal of the Chemistry Society 1704-1710

Mousa, H.A.-L. 1997 Journal of Hospital Infection 37, 317-323

Muller, M. 2002 Free Radical Biology \& Medicine 33, 1527-1533

Muller, M. 2006 Free Radical Biology \& Medicine 41, 1670-1677

Netchiporouk, L.I., Shram, N.F., Jaffrezic-Renault, N., Martelet, C. and Cespuglio, R. 1996

Analytical Chemistry 68, 4358-4364

O'Malley, Y.Q., Reszka, K.J., Spitz, D.R., Denning, G.M, and Britigan, B.E. American Journal of Physiology, Lung Cellular and Molecular Physiology 287, L94-L103

Park, J.-K., Tran, P.H., Chao, J.K.T., Ghodadra, R., Rangarajan, R. and thakor, N.V. 1998

Biosensors and Bioelectronics 13, 1187-1195

Pirnay, J.-P., De Vos, D., Cochez, C., Bilocq, F., Pirson, J., Struelens, M., Duinslaeger, L., Cornelis, P., Zizi, M. abnd Vanderkelen, A. 2003, Journal of Clinical Microbiology 41, 1192-1202

Plowman, R. 2000 Eurosurveillance 5, 49-50

Rasmussen, T.B. and Givskov, M. 2006 Microbiology-SGM 152, 895-904

Reyes, E.A.P., Bale, M.J., Cannon, W.H. and Matsen, J.M. 1982 Journal of Clinical Microbiology $13,456-458$

Schaber, J.A., Carty, N.L., McDonald, N.A., Graham, E.D., Cheluvapa, R., Griswold, J.A. and Hamood, A.N. 2004 Journal of Medical Microbiology 53, 841-853

Schuvailo, O.N., Dzyadevych, S.V., El'skaya, A.V., Gautier-Sauvigne, S., Csoregi, E., Cespuglio, R. and Soldatkin, A.P. 2005 Biosensors and Bioelectronics 21, 87-94

Sharp, D. and Davis, J. Electrochemistry Communications 2008, 10, 708-713

Smith, R.S. and Iglewski, B.H. 2003 Current Opinions in Microbiology 6 56-60

Suard-Chagny, M.-F. 2004 Methods 33, 322-329

Tao, Y., Lin, Z.J., Chem, X.M. and Wang, X.R. 2007 Anal. Chim. Acta 594, 169-174

Tredget, E.E., Shankowsky, H.A., Rennie, R., Burrell, R.E. and Logsetty, S. 2004 Burns 30, 3-26

Usher, L.R., Lawson, R.A., Geary, I., Taylor, C.J., Bingle, C.D., Taylor, G.W. and Whyte, M.K.B. 2002, The Journal of Immunology 168, 1861-1868

Vidhani, S., Mehndiratta, P.L. and Mathur, M.D. 2001 Indian Journal of Medical Microbiology 19, 13-16

Vukomanovik, D.V., Zoutman, D.E., Marks, G.S., Brien, J.F., van Loon, G.W. and Nakatsu, K. 1996 Journal of Pharmacological and Toxicological Methods 36, 97-102

Watson, D., MacDermot, J., Cole, P.J. and Taylor, G.W. European Journal of Biochemistry 150, $309-313$ 
Wilson, R., Sykes, D.A., Watson, D., Rutman, A., Taylor, G.W. and Cole, P.J. 1988 Infection and Immunity. 56, 2515-2517

Yavich, L. and Tiiohen, J. 2000 Journal of Neuroscience Methods 104, 55-63

Figures

Figure 1. (A) Schematic of the lamination process

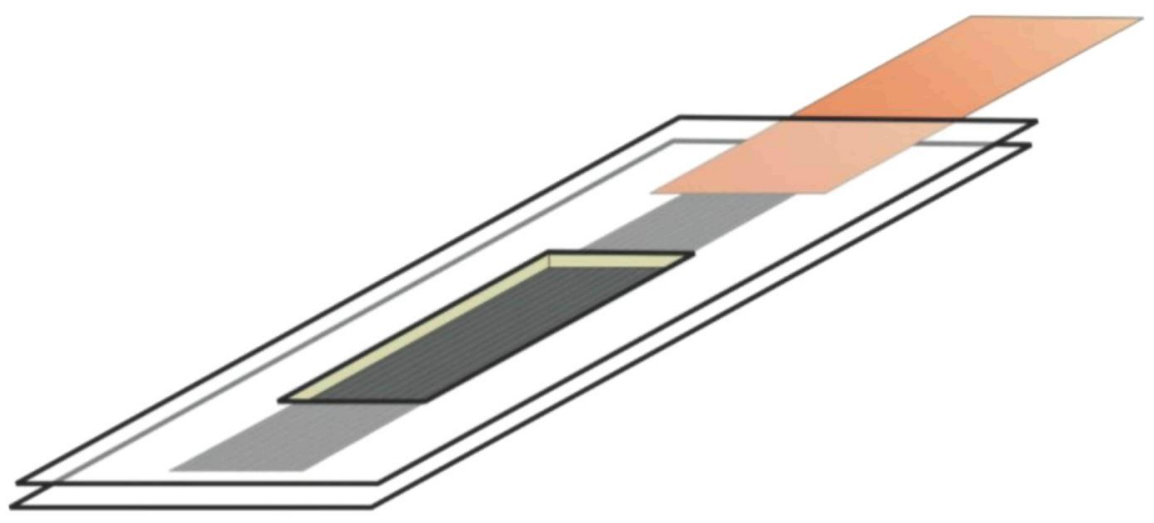

Figure 2. Photochemical reactor setup used for enhanced efficiency Phenazine Methosulfate photooxidation to Pyocyanin
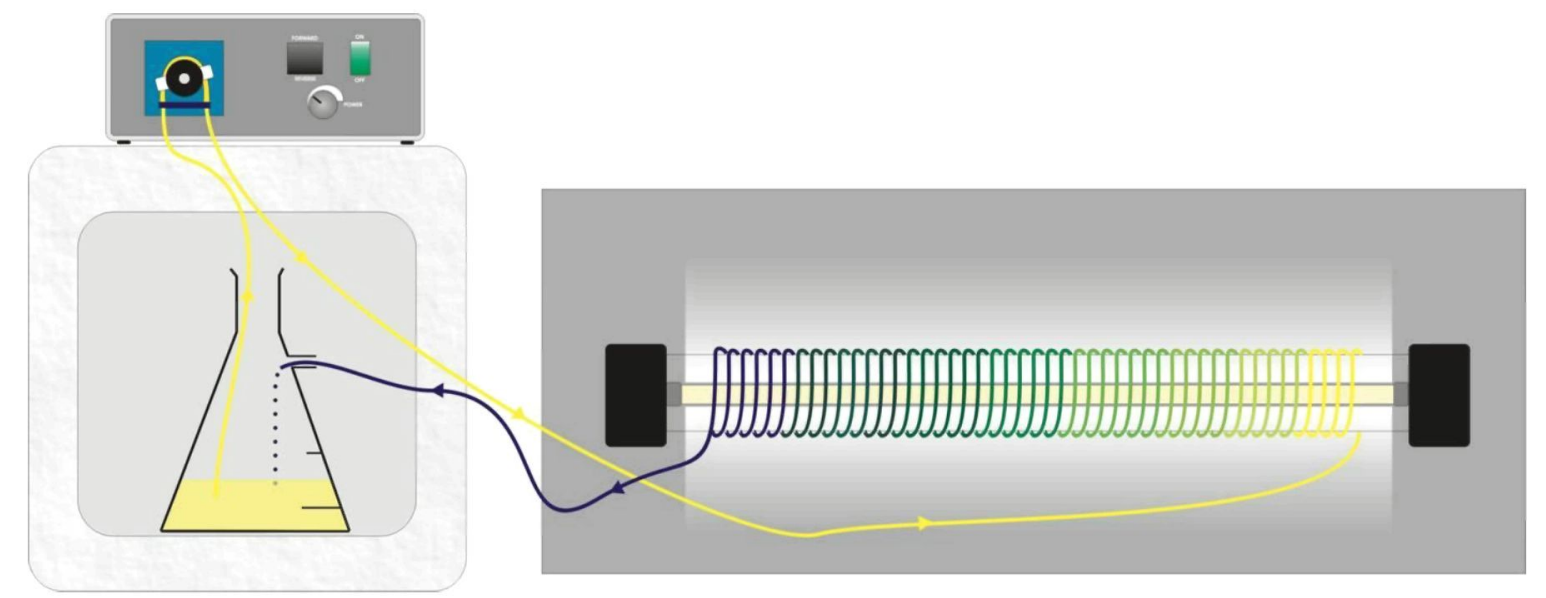